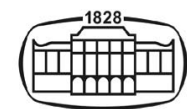

AKADÉMIAI KIADÓ

\title{
Comparing identification of clinically relevant Prevotella species by VITEK MS and MALDI biotyper
}

\section{Acta Microbiologica et Immunologica Hungarica}

(2019), 1-8

DOI: 10.1556/030.66.2019.022

(c) 2019 Akadémiai Kiadó, Budapest

ORIGINAL ARTICLE

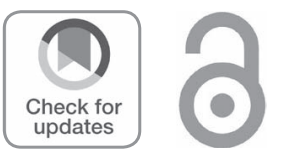

* Corresponding author: Nurver Ulger Toprak Department of Medical Microbiology, School of Medicine, Marmara University, Basıbuyuk Campus, 34854 Maltepe, Istanbul, Turkey Phone: +90 533450 1489; Fax: +90 2164212222

E-mail: nulger@marmara.edu.tr **Members of the ESGAl participated in the study: Eva Leitner (Austria), Suzana C. Stingue, Arne C. Rodloff (Germany), Joseph Paparaskevas (Greece), Wafaa Jamal, Vincent 0. Rotimi (Kuwait), Guven Kulekci, and Hrisi B. Tokman (Turkey).

\section{NURVER ULGER TOPRAK ${ }^{1 *}$, ALIDA C. M. VELOO ${ }^{2}$, EDIT URBAN ${ }^{3}$, INGRID WYBO ${ }^{4}$, HELENE JEAN-PIERRE ${ }^{5}$, TREFOR MORRIS ${ }^{6}$, ULRIK STENZ JUSTESEN ${ }^{7}$, VESNA TRIPKOVIC ${ }^{8}$, SAMO JEVERICA $^{9}$, GUNER SOYLETIR ${ }^{1}$, ELISABETH NAGY ${ }^{3}$ and ON BEHALF OF THE ESCMID STUDY GROUP FOR ANAEROBIC INFECTIONS (ESGAI)**}

\footnotetext{
${ }^{1}$ Department of Medical Microbiology, School of Medicine, Marmara University, Istanbul, Turkey

${ }^{2}$ Department of Medical Microbiology, University Medical Center Groningen, University of Groningen, Groningen, The Netherlands

${ }^{3}$ Institute of Clinical Microbiology, University of Szeged, Szeged, Hungary

${ }^{4}$ Department of Microbiology and Infection Control, Universitair Ziekenhuis Brussel, Brussels, Belgium

${ }^{5}$ Laboratoire de Bactériologie, Hôpital Arnaud de Villeneuve, Centre Hospitalier Régional Universitaire de Montpellier, Montpellier, France

${ }^{6}$ UK Anaerobe Reference Unit, Public Health Wales Microbiology, Cardiff, UK

${ }^{7}$ Department of Clinical Microbiology, Odense University Hospital, Odense, Denmark

${ }^{8}$ Department of Clinical and Molecular Microbiology, University Hospital Center, Zagreb, Croatia

${ }^{9}$ Faculty of Medicine, Institute of Microbiology and Immunology, University of Ljubljana, Ljubljana, Slovenia
}

Received: July 09, 2019 • Accepted: August 12, 2019

\begin{abstract}
In this multicenter study, we aimed to evaluate the performance of MALDI Biotyper and VITEK MS, for identification of Prevotella species. Three hundred and fourteen clinical isolates, collected in eight European countries between January 2014 and April 2016, were identified at the collecting sites by MALDI Biotyper (versions 3.0 and 3.1) and then reidentified by VITEK MS (version 3.0) in the central laboratory. 16S rRNA gene sequencing was used as a standard method. According to sequence analysis, the 314 Prevotella strains belonged to 19 species. MALDI Biotyper correctly identified 281 (89.5\%) isolates to the species level and $33(10.5 \%)$ only at the genus level. VITEK MS correctly identified 253 $(80.6 \%)$ isolates at the species level and $276(87.9 \%)$ isolates at the genus level. Thirty-three isolates belonging to $P$. bergensis, $P$. conceptionensis, $P$. corporis, $P$. histicola, and $P$. nanciensis, unavailable in the VITEK MS 3.0 database, were resulted in genus level or no identification. Six Prevotella strains, belonged to $P$. veroralis, $P$. timonensis, and P. conceptionensis not represented in the MALDI Biotyper system database, were misidentified at the genus level. In conclusion, both VITEK MS and MALDI Biotyper provided reliable and rapid identification. However, the permanent extension of the databases is needed.
\end{abstract}

\section{KEYWORDS}

anaerobic bacteria, Prevotella, 16S rRNA gene sequencing, mass spectrometry, VITEK MS, MALDI Biotyper

\section{INTRODUCTION}

Prevotella species, obligate anaerobic Gram-negative bacilli, are important members of oral, upper respiratory, intestinal, and female genital tract microbiota. However, these organisms 
can also cause opportunistic infections, which may involve any type of oral infection, various types of abscesses, soft tissue, and sterile site infections in the human body. Susceptibility profiles may vary at the species level; consequently, a rapid and an accurate identification of Prevotella isolates plays a critical role in successful treatment $[1,2]$.

The Prevotella genus has undergone recent taxonomic changes and the number of validated species has increased to 50 (http://www.bacterio.net/prevotella.html). The identification of Prevotella isolates classically relies on laborious and timeconsuming phenotypic assays [3]. Nucleic acid sequencing methods have been developed for more rapid and reliable identification. However, DNA-based methods for routine identification of clinical isolates are too expensive, technically complex, labor-intensive, and need expert interpretation of the sequences [4]. Due to the various shortcomings of these methods, there is an increasing interest in more accurate identification of anaerobic bacteria including Prevotella spp. in routine microbiological laboratories.

Previous studies have confirmed that matrix-assisted laser desorption ionization-time-of-flight mass spectrometry (MALDI-TOF MS) is an accurate, rapid, and satisfactory method for routine identification of anaerobes in diagnostic laboratories [5-7]. At present, two MALDI-TOF MS systems are commercially available for routine use: the MALDI Biotyper (Bruker Daltonics Inc., Germany) and VITEK MS (bioMérieux Inc., France). Methodology of the two systems is similar, but differences are present in composition of databases and application of software packages for data analyses [8]. Earlier studies evaluating MALDI-TOF MS identification of Prevotella spp. mostly used the MALDI Biotyper system $[9,10]$.

In a multicenter study organized by ESCMID Study Group for Anaerobic Infections (ESGAI), we assessed the performance of the VITEK MS system for the identification of Prevotella isolates $(n=508)$ collected in 13 countries for a European antibiotic resistance surveillance. We found that VITEK MS offers a reliable and rapid identification of the most frequently isolated Prevotella species [11]. About two third of the isolates were also identified by MALDI Biotyper system in eight participating countries, which made possible to compare the performance of the two MALDI-TOF MS systems. Comparative studies in the field of clinically relevant anaerobic isolates on the applicability of different MS systems are limited. In this study, we describe the identification ability of both MALDI Biotyper and VITEK MS systems for clinically relevant Prevotella species using the $16 \mathrm{~S}$ rRNA gene sequencing as a reference method.

\section{MATERIALS AND METHODS}

\section{Bacterial isolates}

A total of 314 non-duplicate clinically relevant Prevotella isolates were involved in this study. They were a subset of 508 Prevotella strains collected for European antibiotic resistance surveillance with the aim to determine the differences in antibiotic resistance among various species [2]. The 314
Prevotella strains, involved in this study, were collected between January 2014 and April 2016 in eight European countries [Belgium $(n=45)$, Croatia $(n=33)$, Denmark $(n=29)$, France $(n=45)$, Great Britain $(n=41)$, Hungary $(n=47)$, Netherlands $(n=45)$, and Slovenia $(n=29)]$, where mass spectrometry-based identification was already used in the routine practice. All centers involved used the MALDI Biotyper system for identification; however, the software and databases were slightly different in the different collecting sites (Table I). The request was to submit all clinical isolates belonging to genus Prevotella identified by the Bruker Biotyper system. Prevotella isolates were sent to Turkey, in anaerobic transport medium (Anaerobe systems, Morgan Hill, USA). Upon arrival, the isolates were immediately grown on Brucella agar (Difco, USA) supplemented with 5\% sheep blood, hemin, and vitamin $\mathrm{K} 1$ at $36^{\circ} \mathrm{C}$ for $48 \mathrm{~h}$ in an anaerobic chamber (Bactron-I, SHELLAB, USA). Cultures were assessed in terms of purity. Pure and viable strains were stored at $-80{ }^{\circ} \mathrm{C}$ in $10 \%$ skimmed milk until further investigations.

To compare the performance of the two MALDI-TOF MS systems for the identification of Prevotella strains, the isolates were reidentified using the VITEK MS (database version 3.0) in the central laboratory in Turkey. Only isolates that gave species-level identification by the sequencing data were further compared for the MS-based identification.

\section{Identification by MALDI Biotyper MS}

The isolates were identified according to the manufacturer's guidelines. Briefly, a colony was smeared onto a spot on the MALDI plate. The spots were covered with $1 \mu \mathrm{l}$ of matrix solution (Bruker Daltonik $\alpha$-cyano-4-hydroxy-cinnamic acid in $50 \%$ acetonitrile and $2.5 \%$ trifluoroacetic acid) and allowed to air dry. The plate is placed in the ionization chamber of the Biotyper MS machine. The generated mass spectra were compared against a database of mass spectra by the software, resulting in identification of the organism.

All collecting sites used the same criteria for the identification during the collection of the strains. Species-level identification by the MALDI Biotyper was accepted if the $\log$ score was $\geq 2.000$ and genus-level identification if the $\log$ score was between $>1.700$ and $<2.000$. Only isolates with correct genus-level identification were accepted for the study and were sent to the central laboratory.

\section{Identification by VITEK MS}

In case of VITEK MS, according to the manufacturer's guidelines, a single colony was applied onto a spot of target slide as a thin film and allowed to air dry. The spots were covered with $1 \mu \mathrm{l}$ of matrix solution (VITEK MS $\alpha$-cyano-4hydroxycinnamic acid) and allowed to dry at room temperature. Then, the prepared target slide was inserted into the VITEK MS machine (bioMérieux). Microbial identification was performed by generating spectra from the bacterial extracts and comparing them with the reference spectra using the VITEK MS version 3.0 database. 
Table I. Number of Prevotella strains collected in different countries and the MALDI Biotyper software and database used for their identification at the different collection sites

\begin{tabular}{|c|c|c|c|c|c|}
\hline \multirow[b]{2}{*}{$\begin{array}{l}\text { Collecting sites } \\
\text { (no. of isolates) }\end{array}$} & \multirow{2}{*}{$\begin{array}{c}\text { No. of strains } \\
\text { identified on } \\
\text { species/genus level } \\
\text { by Biotyper }\end{array}$} & \multirow{2}{*}{$\begin{array}{c}\text { No. of different } \\
\text { Prevotella spp. } \\
\text { identified by Biotyper } \\
\text { at the collection sites }\end{array}$} & \multicolumn{3}{|c|}{ Data about the Biotyper used } \\
\hline & & & Software & Database & $\begin{array}{l}\text { No. of Prevotella } \\
\text { spp./MSPs in the } \\
\text { database }\end{array}$ \\
\hline Belgium (45) & $41 / 4$ & 14 & 3.0 & 5,989 & $31 / 77^{b}$ \\
\hline Croatia (33) & $33 / 0$ & 6 & 3.1 & 5,989 & $31 / 77$ \\
\hline Denmark (29) & $28 / 1$ & 9 & 3.1 & 6,903 & $31 / 126$ \\
\hline France (45) & $40 / 5$ & 12 & 3.0 & 5,627 & $23 / 53$ \\
\hline Great Britain (41) & $41 / 0$ & 8 & 3.0 & 5,989 & $31 / 77$ \\
\hline Hungary (47) & $46 / 1$ & 9 & 3.0 & 5,627 & $23 / 53$ \\
\hline The Netherlands (45) & $45 / 0$ & 12 & 3.0 & 4,613 & $23 / 52$ \\
\hline Slovenia (29) & $29 / 0$ & 12 & 3.0 & 5,627 & $23 / 53$ \\
\hline
\end{tabular}

Note: MALDI: matrix-assisted laser desorption ionization; MSPs: main spectra profiles.

${ }^{a}$ Species-level identification was accepted if $\log$ score was $\geq 2.000$ and genus-level identification was accepted if log score was $\geq 1.700-<2.000$.

II this center, an extended database was used according to Wybo et al. [9].

Species-level identification was considered accurate, if confidence level was $\geq 99.9 \%$ and for a confidence level ranging between $60 \%$ and $99.9 \%$ with a single species choice. Low-level confidence score $(<50 \%)$ is found in a low discrimination identification consisting of a list of two to four choices for an identification match. These isolates were identified only genus level. The bacterium was considered non-identified, if confidence level value was $<60 \%$ and no match for the composite spectra. These criteria were used for the identification of the isolates in the central laboratory.

\section{Analysis of the MALDI-TOF MS data}

MALDI-TOF MS identification data by the two systems obtained in the collecting laboratories and in the central laboratory were compared with the identification results obtained by sequencing and the data were categorized as follows: (1) correct identification at the species level; (2) correct identification at the genus level, including different species within the Prevotella genus; (3) misidentification (minor error: different Prevotella species; major error: different genus); and (4) no identification.

\section{S rRNA gene sequencing}

A "gold standard" $16 \mathrm{~S}$ rRNA gene sequencing of all isolates was carried out in the central laboratory in Turkey using the methodology described by Song et al. [12]. Sequences were analyzed using GenBank (www.ncbi.nlm.nih.gov). Identification at species level was accepted in the presence of $\geq 99.0 \%$ similarity with the $16 \mathrm{~S}$ rRNA gene sequence and $97.0 \%-99.0 \%$ similarity at the genus level [13].

\section{RESULTS}

All 314 Prevotella isolates were identified at the species level with $\geq 99.0 \%$ sequence similarity using the $16 \mathrm{~S}$ RNA gene sequencing. A total of 19 different Prevotella species were identified, most of which were P. bivia $(n=74)$, followed by P. buccae $(n=41)$. $P$. nigrescens and $P$. denticola were represented equally ( 34 isolates each), but some of the species were present only in very low numbers ( $\leq 4$ isolates), such as $P$. buccalis, $P$. conceptionensis, $P$. corporis, $P$. histicola, $P$. salivae, and $P$. veroralis. Table II shows the distribution of the Prevotella spp. identified by the 16S RNA gene sequencing and the identification results obtained by the MALDI Biotyper at the isolation sites applying different databases (Table I) and by the VITEK MS in the central laboratory. Concordant species-level identification between the three identification systems was measured; 16S rRNA gene sequencing, MALDI Biotyper, and VITEK MS gave the same species-level identification in $76.4 \%(n=240)$ of the isolates (Table II). There were discrepancies (minor or major errors) compared with the sequencing data for the remaining 74 (23.6\%) isolates. Discrepant results are shown in Table III.

Comparing the sequencing data with the identification obtained by the MALDI Biotyper using different databases at the collecting sites, $281(89.4 \%)$ of the 314 isolates were identified correctly at the species level and 33 isolates were only correctly identified at genus level showing different species (minor error) or only being identified as Prevotella spp. (Table III). Most of the misidentified organisms belonged to P. bivia, P. buccae, $P$. denticola, and P. timonensis, but many other species were misidentified by showing 
Table II. The distribution of Prevotella species according to the sequencing data and identification results obtained by MALDI Biotyper and VITEK MS

\begin{tabular}{|c|c|c|c|c|c|c|}
\hline \multicolumn{2}{|c|}{ 16S rRNA gene sequencing } & \multicolumn{2}{|c|}{$\begin{array}{l}\text { MALDI Biotyper used at the } \\
\text { collecting sites }\end{array}$} & \multicolumn{2}{|c|}{ VITEK MS used at the central laboratory } & \multirow{2}{*}{$\begin{array}{l}\text { Concordant } \\
\text { species } \\
\text { identification by } \\
\text { the two MS } \\
\text { systems }(n)\end{array}$} \\
\hline Prevotella species & $n(\%)$ & $\begin{array}{c}\text { Correct species ID } \\
(n)\end{array}$ & $\begin{array}{l}\text { Correct genus ID } \\
(n)\end{array}$ & $\begin{array}{c}\text { Correct species ID } \\
(n)\end{array}$ & $\begin{array}{c}\text { Correct genus ID } \\
(n)\end{array}$ & \\
\hline P. baroniae & $13(4.1)$ & 12 & 13 & 11 & 12 & 11 \\
\hline P. bergensis ${ }^{\mathrm{a}}$ & $12(3.8)$ & 10 & 12 & 0 & 1 & 0 \\
\hline P. bivia & $74(23.6)$ & 69 & 74 & 74 & 74 & 69 \\
\hline P. buccae & $41(13.1)$ & 36 & 41 & 38 & 38 & 36 \\
\hline P. buccalis & $4(1.3)$ & 3 & 4 & 4 & 4 & 3 \\
\hline P. conceptionensis ${ }^{\mathrm{a}, \mathrm{b}}$ & $1(0.3)$ & 0 & 1 & 0 & 0 & 0 \\
\hline P. corporis $^{\mathrm{a}}$ & $4(1.3)$ & 3 & 4 & 0 & 1 & 0 \\
\hline P. denticola & $34(10.8)$ & 30 & 34 & 32 & 34 & 30 \\
\hline P. disiens & $18(5.7)$ & 17 & 18 & 17 & 17 & 17 \\
\hline P. histicola ${ }^{\mathrm{a}}$ & $4(1.3)$ & 3 & 4 & 0 & 4 & 0 \\
\hline P. intermedia & $9(2.9)$ & 9 & 9 & 7 & 8 & 7 \\
\hline P. melaninogenica & $27(8.6)$ & 25 & 27 & 25 & 26 & 25 \\
\hline P. nanceiensis ${ }^{\mathrm{a}}$ & $12(3.8)$ & 12 & 12 & 0 & 2 & 0 \\
\hline P. nigrescens & $34(10.8)$ & 33 & 34 & 24 & 29 & 24 \\
\hline P. oralis & $5(1.6)$ & 3 & 5 & 5 & 5 & 3 \\
\hline P. oris & $8(2.5)$ & 8 & 8 & 8 & 8 & 8 \\
\hline P. salivae & $4(1.3)$ & 3 & 4 & 2 & 4 & 2 \\
\hline P. timonensis & $8(2.5)$ & 4 & 8 & 4 & 7 & 4 \\
\hline P. veroralis & $2(0.6)$ & 1 & 2 & 2 & 2 & 1 \\
\hline Total [n (\%)] & $314(100)$ & $281(89.4)$ & $314(100)$ & $253(80.6)$ & $276(87.9)$ & $240(76.4)$ \\
\hline
\end{tabular}

Note: Concordant species identification by the two MALDI-TOF MS systems is also included. MALDI: matrix-assisted laser desorption ionization; MS: mass spectrometry.

${ }^{a}$ Not included in the database of the VITEK MS system during the investigation.

other species as well. The MALDI Biotyper database versions DB 4613 and DB 5627 did not contain P. conceptionensis, $P$. timonensis, or $P$. veroralis; therefore, six isolates evaluated by these data sets were only identified at the genus level by the MALDI Biotyper (Table III).

Comparative analysis of the identification obtained by the VITEK MS system and the 16S RNA gene sequencing of the isolates revealed that VITEK MS correctly identified 253 isolates $(80.6 \%)$ at the species level and $276(87.9 \%)$ at the genus level (Table II) and misidentified 27 isolates with minor error $(n=23)$ or major error $(n=4$; Table III). A total of 34 isolates $(10.8 \%)$ were not identified. Thirty-three isolates from five Prevotella species (P. bergensis, $P$. conceptionensis, $P$. corporis, $P$. histicola, and $P$. nanciensis) were not available in the VITEK MS IVD 3.0 database, and were not identified or misidentified as different Prevotella species or different genus (Tables II and III).

\section{DISCUSSION}

Recently, in microbiological diagnostic laboratories, there is an increased usage of the MALDI-TOF MS technique for rapid identification of clinically significant bacteria including anaerobes [8]. Various studies indicated that the performance of the MALDI-TOF MS systems is highly 


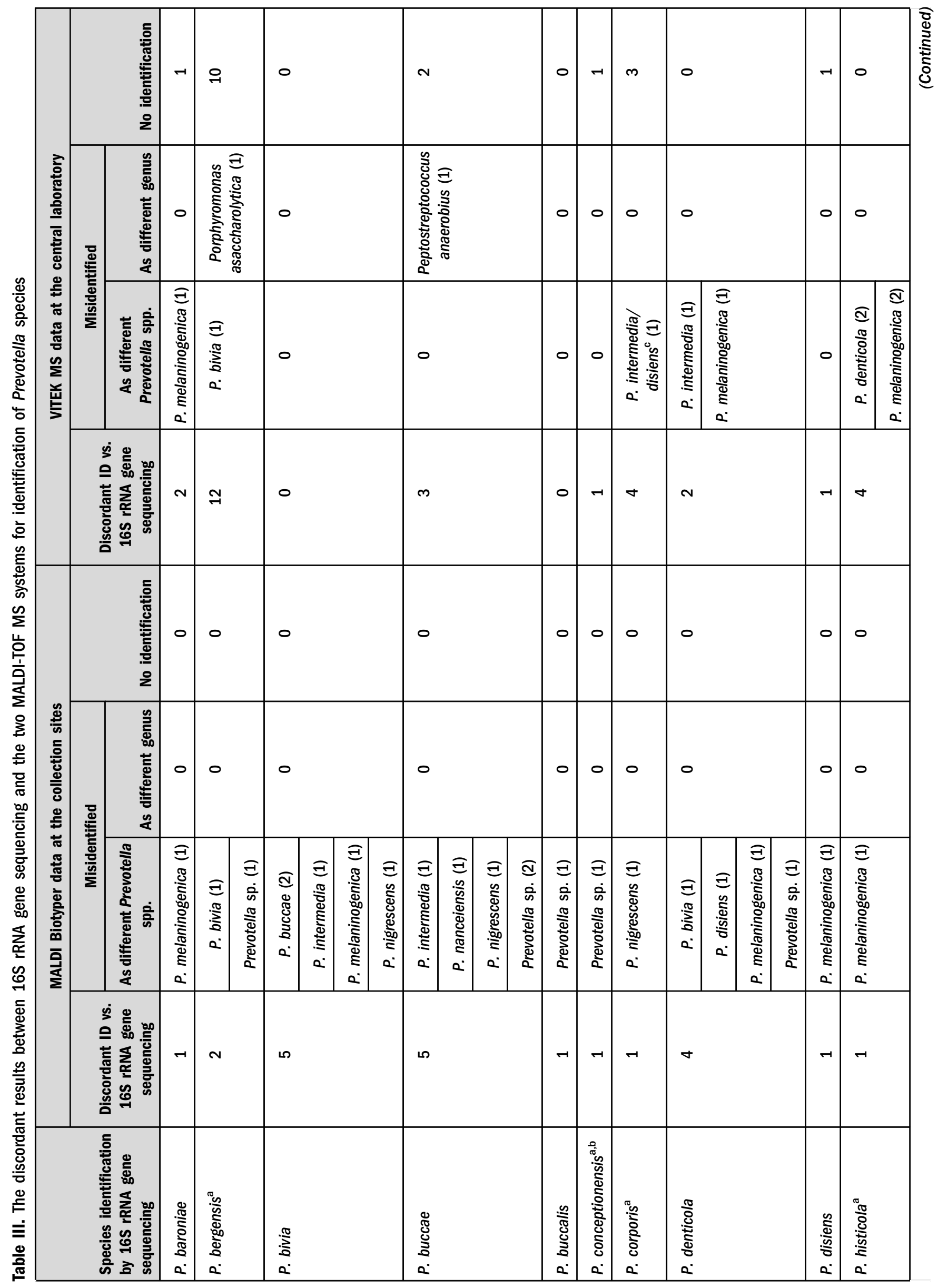




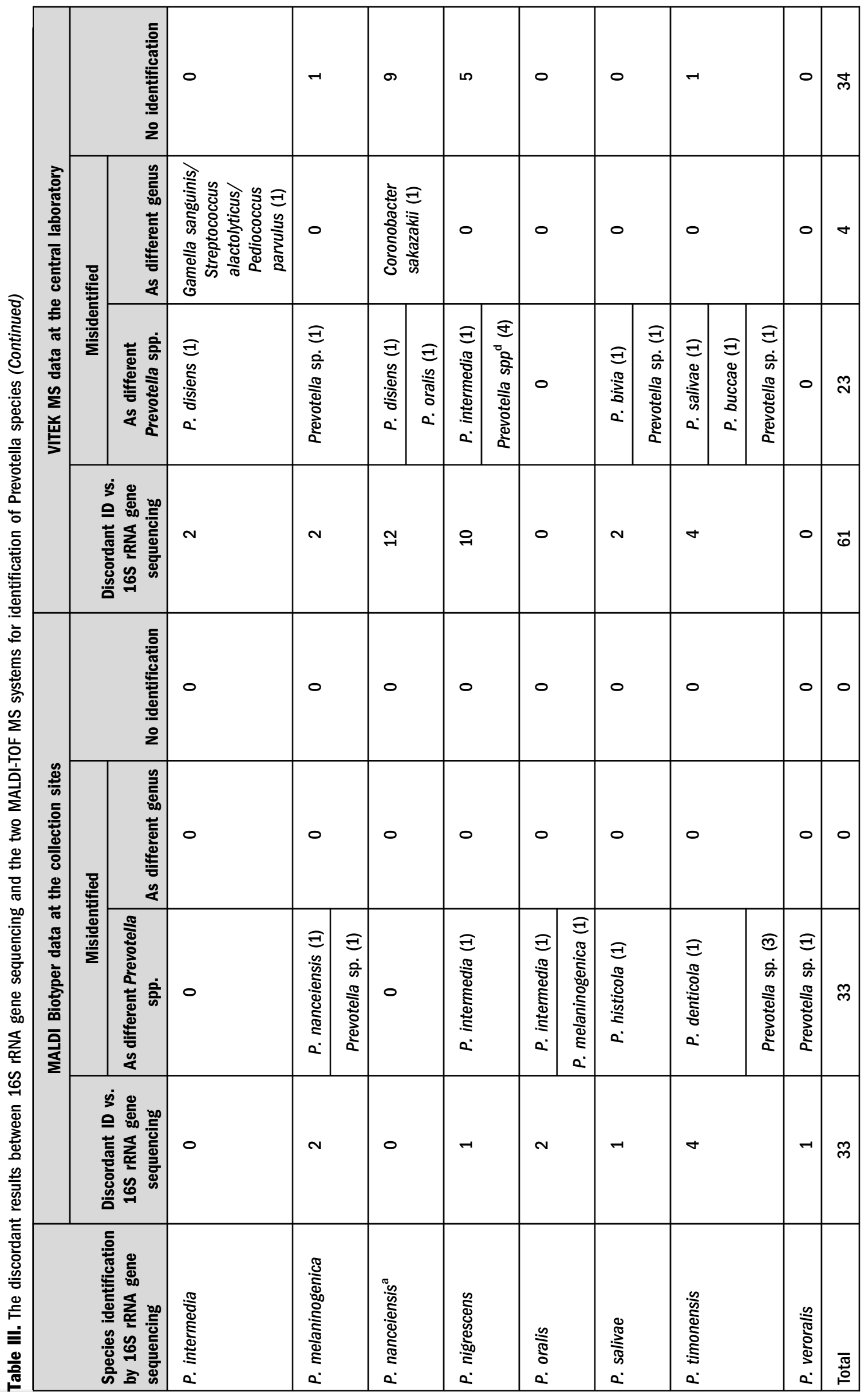

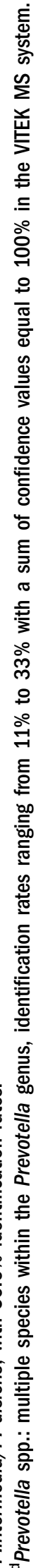


dependent on the expansion of the accompanying databases and the specific group of isolates studied [4-7, 14]. Recent reports show that the MALDI-TOF MS can provide identification for $>90 \%$ of anaerobes $[5,10]$, whereas initial studies put this number at $<70 \%[7,15]$. The inclusion of sequenced clinical isolates to the databases of the MALDI-TOF systems has also been suggested $[9,16]$.

To support this trend, in this multicenter study, we compared the applicability of two MALDI-TOF MS systems for the identification of Prevotella species and compared the results with $16 \mathrm{~S}$ rRNA gene sequencing data. The results showed that the performances of VITEK MS and MALDI Biotyper were highly accurate for species identification (80.6\% and $89.4 \%$, respectively) and were even better for genus-level identification ( $87.9 \%$ and $100 \%$, respectively). However, 33 isolates from five different Prevotella species, such as $P$. bergensis, $P$. conceptionensis, $P$. corporis, $P$. histicola, and $P$. nanciensis, were not identified or misidentified by the current VITEK MS database version 3.0, which does not cover these Prevotella species. Out of the different databases used for the MALDI Biotyper during this study, some species of Prevotella (P. concepcionensis, $P$. veroralis, and $P$. timonensis) were also unavailable, which also influenced the identification accuracy of the system.

Previous studies on the identification of anaerobic bacteria with MALDI-TOF MS used only a few Prevotella isolates representing limited number of species [5, 15, 17-20]. All those earlier studies that focused for the identification of Prevotella species used the MALDI Biotyper in comparison with $16 \mathrm{~S}$ rRNA gene sequencing. The first of these studies was published by Wybo et al. [9] and was conducted in 2012 . This study tested 102 clinical isolates belonging to different species of Prevotella of which only $62.7 \%$ were identified at the species level and $73.5 \%$ at the genus level. Accurate species and genus identification went up to $83.3 \%$ and $89.2 \%$, respectively, when the commercial database was extended with in-house reference spectra from 23 sequenced Prevotella reference strains and 7 clinical isolates. The second study, Gursoy et al. [10] in 2017, used 123 oral Prevotella isolates to test the diagnostic accuracy of the MALDI Biotyper. This study yielded $88.6 \%$ accuracy at the species level and $100 \%$ accuracy at the genus level. The most likely reason for this increase in accuracy in the past 5 years is the expanded database of the MALDI Biotyper covering more Prevotella spp. The database used by Wybo et al. [9] contained 20 Prevotella species, whereas the database for the 2017 study by Gursoy et al. [10] contained 30. The results of this study $(89.2 \%$ of species-level identification of 314 isolates), obtained using MALDI Biotyper system with different main spectra profiles of 23-31 Prevotella spp. in the databases used in the collecting laboratories, are similar to those ones obtained by Gursoy et al. [10].

The first study evaluating how well the VITEK MS (database version 2.0) identifies anaerobic bacteria on a multicenter level was conducted by Garner et al. [21]. This study tested a total of 652 anaerobic clinical isolates, including 90 isolates from five different Prevotella species (P. bivia,
P. buccae, P. denticola, P. intermedia, and P. melaninogenica). Of these strains, $91.1 \%$ were identified at the species level. In this study, using the VITEK MS database version 3.0, 95.1\% of the Prevotella strains belonging to the same species were correctly identified at the species level. The overall lower percentage of the species-level identification by the VITEK MS achieved in this study evaluating all the isolates may be due to the much higher number of species represented. The strains used in this study belonged to 19 different species, including species not present in the current VITEK MS database.

There are limited number of studies, which compare the performance of the two commercially available MALDI-TOF MS systems especially for the identification of special anaerobic bacteria such as Prevotella. The limitation of conducting this type of research is the relatively low number of Prevotella species in the VITEK MS database (16 species at the moment), compared with the MALDI Biotyper databases, which contained reference spectra for 23 species (DB 4613 and DB 5627) and 31 species (DB 5989 and DB 6903) used in this study. However, this study also shows that, besides the number of the species present in the databases, many other factors such as incubation time, sample preparation, or maintenance of the system (which were not tested during this study) may influence the correct species-level identification as well [22].

The strengths of this study are the wide range of clinically relevant Prevotella spp. tested and the use of 16S rRNA gene sequencing as the reference method. However, there were some limitations of this study such as the identification of the isolates by the two MALDI-TOF MS systems was not carried out in the same laboratory from the same culture plate simultaneously. Furthermore, the collecting sites used different MALDI Biotyper databases for the original identification of the isolates and we have not collected data whether the sample preparation was the same in all sites where the MALDI Biotyper was used. Several database developments for both systems were carried out, since we closed down this study [16].

In conclusion, both MALDI-TOF MS systems performed well in identifying most clinically important Prevotella strains. MALDI-TOF MS is a reliable alternative to $16 \mathrm{~S}$ rRNA gene sequencing for these species, considered to be the gold standard for identification of bacteria. Moreover, it is easier and more applicable in routine laboratories. However, expanding and optimizing the MS databases to include reference spectra for more species is needed. Controlled sample preparation and careful evaluation of the cut-off values to accept species determination are advisable. Accurate and simple identification of isolates will increase interest in studying anaerobic organisms, enhancing our knowledge of the epidemiology, pathogenicity, and clinical relevance of Prevotella species.

Acknowledgements: The authors would like to thank Mike Cox for providing transport medium. They would also like to thank Sedef Glover for editing the manuscript. The study was funded in part by ESGAI Research Grant. 
Conflict of Interest: The authors declare no conflict of interest.

Ethical approval: The study was approved by the Ethics Committee of Marmara University (no. 09.2013.0248). No informed consent required as this study did not involve human subjects or samples.

\section{REFERENCES}

1. Könönen, E., Wade, W. G., Citron, D. M.: Bacteroides, porphyromonas, Prevotella, Fusobacterium, and other anaerobic Gram-negative rods. In Versalovic, J., Carroll, K. C., Jorgensen, J. H., Funke, G., Landry, M. L., Warnock, D. W. (eds): Manual of Clinical Microbiology, Volume 2, $10^{\text {th }}$ Edition. ASM Press, Washington, DC, 2011, pp. 858-880.

2. Ulger Toprak, N., Veloo, A. C. M., Urban, E., Wybo, I., Justesen, U. S., Jean-Pierre, H., Morris, T., Akgul, O., Kulekci, G., Soyletir, G., Nagy, E., ESCMID Study Group for Anaerobic Infections (ESGAI): A multicenter survey of antimicrobial susceptibility of Prevotella species as determined by Etest methodology. Anaerobe 52, 9-15 (2018).

3. Jusimies-Somer, H., Summanen, P., Citron, D. M., Baron, E. J., Wexler, H. M., Finegold, S. M.: Wadsworth-KTL Anaerobic Bacteriology Manual, $6^{\text {th }}$ Edition. Star Publishing, Belmont, CA, 2002, pp. 86-98.

4. Yunoki, T., Matsumura, Y., Nakano, S., Kato, K., Hotta, G., Noguchi, T., Yamamoto, M., Nagao, M., Takakura, S., Ichiyama, S.: Genetic, phenotypic and matrix-assisted laser desorption ionization time-of-flight mass spectrometry-based identification of anaerobic bacteria and determination of their antimicrobial susceptibility at a university hospital in Japan. J Infect Chemother 22, 303-307 (2016).

5. Rodríguez-Sánchez, B., Alcalá, L., Marín, M., Ruiz, A., Alonso, E., Bouza, E.: Evaluation of MALDI-TOF MS (matrix-assisted laser desorption-ionization time-of-flight mass spectrometry) for routine identification of anaerobic bacteria. Anaerobe 42, 101-107 (2016).

6. Wilson, D. A., Young, S., Timm, K., Novak-Weekley, S., Marlowe, E. M., Madisen, N., Lillie, J. L., Ledeboer, N. A., Smith, R., Hyke, J., Griego-Fullbright, C., Jim, P., Granato, P. A., Faron, M. L., Cumpio, J., Buchan, B. W., Procop, G. W.: Multicenter evaluation of the Bruker MALDI Biotyper CA system for the identification of clinically important bacteria and yeasts. Am J Clin Pathol 147, 623-631 (2017).

7. Shannon, S., Kronemann, D., Patel, R., Schuetz, A. N.: Routine use of MALDI-TOF MS for anaerobic bacterial identification in clinical microbiology. Anaerobe 53, 191-196 (2018).

8. Patel, R.: MALDI-TOF MS for the diagnosis of infectious diseases. Clin Chem 61, 100-111 (2015).

9. Wybo, I., Soetens, O., De Bel, A., Echahidi, F., Vancutsem, E., Vandoorslaer, K., Piérard, D.: Species identification of clinical Prevotella isolates by matrix-assisted laser desorption ionization-time of flight mass spectrometry. J Clin Microbiol 50, 1415-418 (2012).

10. Gürsoy, M., Harju, I., Matomäki, J., Bryk, A., Könönen, E.: Performance of MALDI-TOF MS for identification of oral Prevotella species. Anaerobe 47, 89-93 (2017).
11. Ulger Toprak, N., Veloo, A. C. M., Urban, E., Wybo, I., Justesen, U. S., Jean-Pierre, H., Morris, T., Akgul, O., Kulekci, G., Soyletir, G., Nagy, E., ESCMID Study Group for Anaerobic Infections (ESGAI): Performance of mass spectrometric identification of clinical Prevotella species using the VITEK MS system: A prospective multi-center study. Anaerobe 54, 205-209 (2018).

12. Song, Y., Liu, C., McTeague, M., Finegold, S. M.: 16S ribosomal DNA sequence-based analysis of clinically significant Grampositive anaerobic cocci. J Clin Microbiol 41, 1363-1369 (2003).

13. Woo, P. C., Lau, S. K., Teng, J. L., Tse, H., Yuen, K.: Then and now: Use of $16 \mathrm{~S}$ rDNA gene sequencing for bacterial identification and discovery of novel bacteria in clinical microbiology laboratories. Clin Microbiol Infect 14, 908-934 (2008).

14. Ferrand, J., Bonnet, I., Alauzet, C., Lozniewski, A.: Evaluation of the Vitek MS and the MALDI Biotyper systems for the identification of less commonly isolated but clinically relevant anaerobes and facultative anaerobes. Anaerobe 54, 210-216 (2018).

15. Veloo, A. C., Knoester, M., Degener, J. E., Kuijper, E. J.: Comparison of two matrix-assisted laser desorption ionisationtime of flight mass spectrometry methods for the identification of clinically relevant anaerobic bacteria. Clin Microbiol Infect 17, 1501-1506 (2011).

16. Veloo, A. C. M., Jean-Pierre, H., Justesen, U. S., Morris, T., Urban, E., Wybo, I., Kostrzewa, M., Friedrich, A. W., on behalf of the ENRIA workgroup: Validation of MALDI-TOF MS biotyper database optimized for anaerobic bacteria: The ENRIA project. Anaerobe 54, 224-230 (2018).

17. Lee, W., Kim, M., Yong, D., Jeong, S. H., Lee, K., Chong, Y.: Evaluation of VITEK mass spectrometry (MS), a matrix-assisted laser desorption ionization time-of-flight MS system for identification of anaerobic bacteria. Ann Lab Med 35, 69-75 (2015).

18. Jamal, W. Y., Shahin, M., Rotimi, V. O.: Comparison of two matrix-assisted laser desorption/ionization-time of flight (MALDI-TOF) mass spectrometry methods and API 20AN for identification of clinically relevant anaerobic bacteria. J Med Microbiol 62, 540-544 (2013).

19. Nagy, E., Backer, S., Kostrzewa, M., Barta, N., Urban, E.: The value of MALDI-TOF MS for the identification of clinically relevant anaerobic bacteria in routine laboratories. J Med Microbiol 61, 1393-1400 (2012).

20. Coltella, L., Mancinelli, L., Onori, M., Lucignano, B., Menichella, D., Sorge, R., Raponi, M., Mancini, R., Russo, C.: Advancement in the routine identification of anaerobic bacteria by MALDI-TOF mass spectrometry. Eur J Clin Microbiol Infect Dis 32, 1183-1192 (2013).

21. Garner, O., Mochon, A., Branda, J., Burnham, C. A., Bythrow, M., Ferraro, M., Ginocchio, C., Jennemann, R., Manji, R., Procop, G. W., Richter, S., Rychert, J., Sercia, L., Westblade, L., Lewinski, M.: Multi-center evaluation of mass spectrometric identification of anaerobic bacteria using the VITEK $^{\infty}$ MS system. Clin Microbiol Infect 20, 335-339 (2014).

22. Veloo, A. C. M., Elgarsma, P. E., Fridrich, A. W., Nagy, E., von Winkelhoff, A. J.: The influence of incubation time, sample preparation and exposure to oxygen on the quality of MALDITOF MS spectrum of anaerobic bacteria. Clin Microbiol Infect 20, O1091-O1097 (2014). 